\title{
Advanced predictive quality control strategy involving different facilities
}

\author{
Joaquín Ordieres-Meré • Ana González-Marcos • \\ Fernando Alba-Elías · César Menéndez-Fernández
}

\begin{abstract}
There are many industries that use highly technological solutions to improve quality in all of their products. The steel industry is one example. Several automatic surface-inspection systems are used in the steel industry to identify various types of defects and to help operators decide whether to accept, reroute, or downgrade the material, subject to the assessment process. This paper focuses on promoting a strategy that considers all defects in an integrated fashion. It does this by managing the uncertainty about the exact position of a defect due to different process conditions by means of Gaussian additive influence functions. The relevance of the approach is in making possible consistency and reliability between surface inspection systems. The results obtained are an increase in confidence in the automatic inspection system and an ability to introduce improved
\end{abstract}

prediction and advanced routing models. The prediction is provided to technical operators to help them in their decision-making process. It shows the increase in improvement gained by reducing the $40 \%$ of coils that are downgraded at the hot strip mill because of specific defects. In addition, this technology facilitates an increase of $50 \%$ in the accuracy of the estimate of defect survival after the cleaning facility in comparison to the former approach. The proposed technology is implemented by means of software-based, multi-agent solutions. It makes possible the independent treatment of information, presentation, quality analysis, and other relevant functions.

Keywords Advanced quality strategy •

Multi-agent-based quality information system . Surface defects monitoring - Comparison of defects along different facilities - Surface defects in steel industry $\cdot$ Artificial intelligence techniques

\section{Introduction}

Classical product production strategies in many industries involve different production steps in different plant facilities where defects can persist from one facility to the next and even to the final product. In many cases, because the geometry of the product in the process of transformation changes, it is necessary for each facility to have robust quality requirements.

This is a conservative approach. A factor mitigating its use is the requirement for a large batch size in order to produce the customer's complete order due to the initial estimate of material that will be rerouted or downgraded. Normally, this results in finishing the 
Fig. 1 Product evolution from slab to the final coil
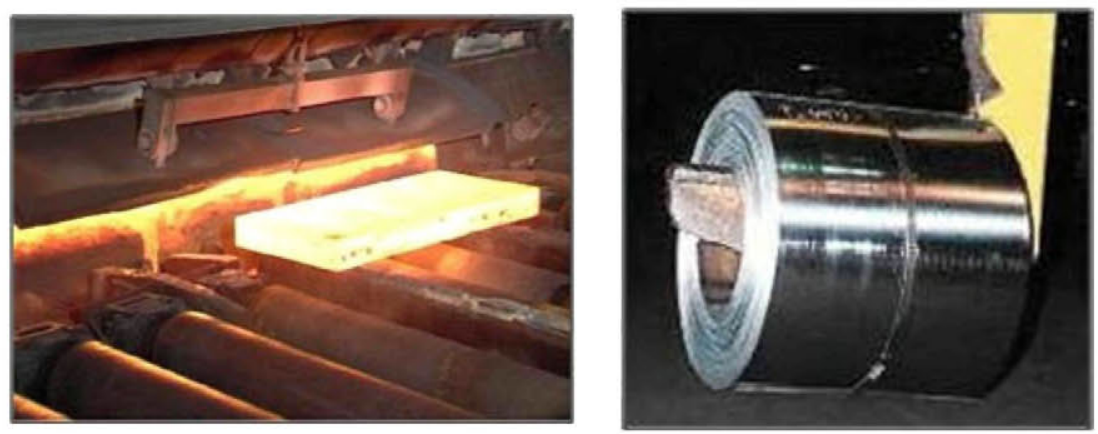

order with unused products left over that, in the end, will have increased the final cost of the batch. The advantage is that step by step quality criteria are more robust. However, no quality prediction is carried out before the checkpoint. Thus, material that has a high probability of being discharged sometimes is routed through expensive processes, and batches may not be completed if there is excessive downgrading due to the operating conditions at specific facilities.

The main limitation to considering defaults globally across facilities was the difficulty in tracking the position of defaults because geometrical transformations made it difficult to develop a defect history. Another difficulty is related to the harmonization of defect sensors across different facilities. Failing to obtain a good degree of consistency makes it possible for some defects to arise for no apparent reason after processes that were not responsible for them, just because sensor's threshold values are lower than before.

This work seeks to offer a holistic approach to the problem by integrating different strategies and technologies to address both of these difficulties. It makes possible the implementation of a softer strategy through several facilities in such a way that evolution of a predictive mechanism for the number of defects becomes affordable.

Without loss of generalization, details will be provided in connection with a specific industry (i.e., the steel industry), as this makes it easier to identify all relevant aspects introduced previously. Similar applications can be found in the aluminum and paper industries, among others.
Fig. 2 Example for some classes of surface defects

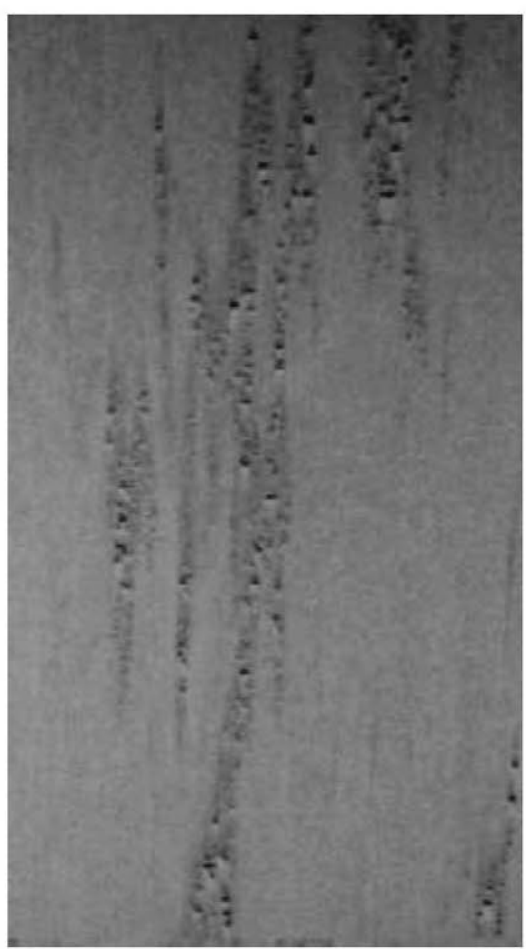

(a) Scale Type C3

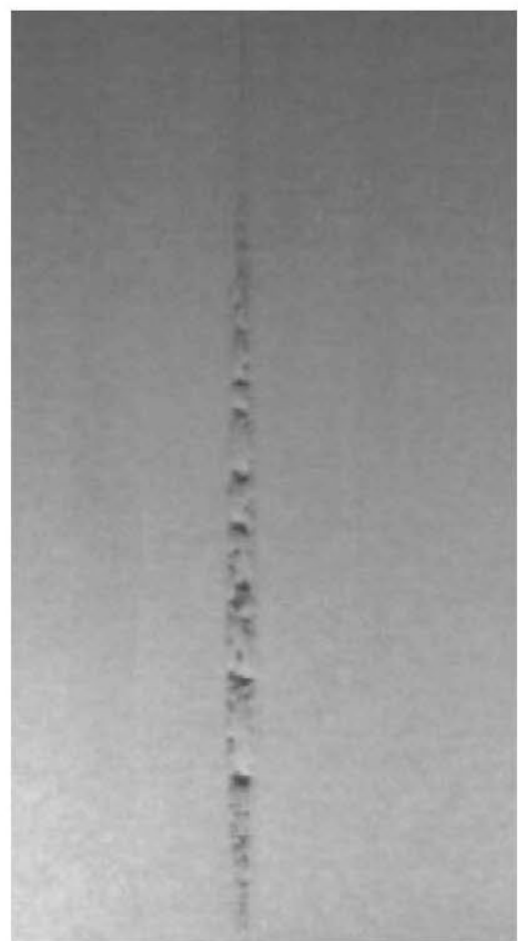

(b) Scale Type C5 
For purposes of clarity, let us focus on the production of steel coils (see Fig. 1) and, as an example, on one specific class of surface defect such as scale defects.

Several facilities, such as the hot strip mill (HSM), the pickling line (PKL), and the hot-dip galvanizing line (HDG) are involved in these types of situations (see Fig. 4). Scale defects on a strip arise in the HSM where an automatic surface-inspection system (ASIS) will provide a defect map (see Fig. 2). The PKL is responsible for cleaning the coil (see Fig. 3), subject to various operating parameters, and taking into account production stream constraints. Those defects can survive the zinc coating at the HDG. Consequently, at the end of the process, the coil must be downgraded.

Potential industrial advantages of producing such a quality-predictive system include an early estimate of defect survival. This means that plant managers will have additional information about the probability of defect persistence depending on the operating conditions of downstream facilities. They will be able to reroute materials, avoid increasing the processing costs by the loss of material being downgraded later, or can simply put aside this material for processing under different operating conditions in those facilities. In this case, it probably will require more extensive rework.
The organization of the paper is as follows. In Section 2 the state of the art will be reviewed, including contributions to defect detection as well as to model development that is based on statistical methods. Section 3 examines surface defect modeling and its application to estimation of defect evolution as well as to providing robustness to the system by comparing the levels of specific existing defects across facilities. Section 4 will discuss its application to an advanced product downgrading strategy. The main conclusions are presented in Section 5.

\section{State of the art}

In recent years, the steel industry has experienced a sharp increase in the quality requirements of steel used in the fabrication of various products. In particular, this pertains to steel from the hot strip mill to the finishing lines. It is especially true for strip that is intended for high-end applications, such as those found in the automotive industry and, to some extent, the packaging materials industry.

In the early years of ASIS, the main topic discussed by users was the tuning of their systems to improve
Fig. 3 Sample map coil at different facilities, including length reduction because of cooling
COIL CODE: $11115034(0)$

Surface \% (Side 0): $\mathrm{HSM}=0.67 \mathrm{PICK}=0.01 \mathrm{C5}=0.00 \mathrm{CC} 5=0.00 \mathrm{CS}+\mathrm{CPR}=0.67 \quad \mathrm{CC} 3+\mathrm{CC} 7=0.01$ Surface \% (Side 1): $\mathrm{HSM}=0.00 \quad \mathrm{PICK}=0.00 \quad \mathrm{C} 5=0.00 \quad \mathrm{CC} 5=0.00 \quad \mathrm{CS}+\mathrm{CPR}=0.00 \quad \mathrm{CC} 3+\mathrm{CC} 7=0.00$

- Scale C5 " Scale CS " Scale CPR

HSM (Side 0)

Length $=910 \mathrm{~m}$.

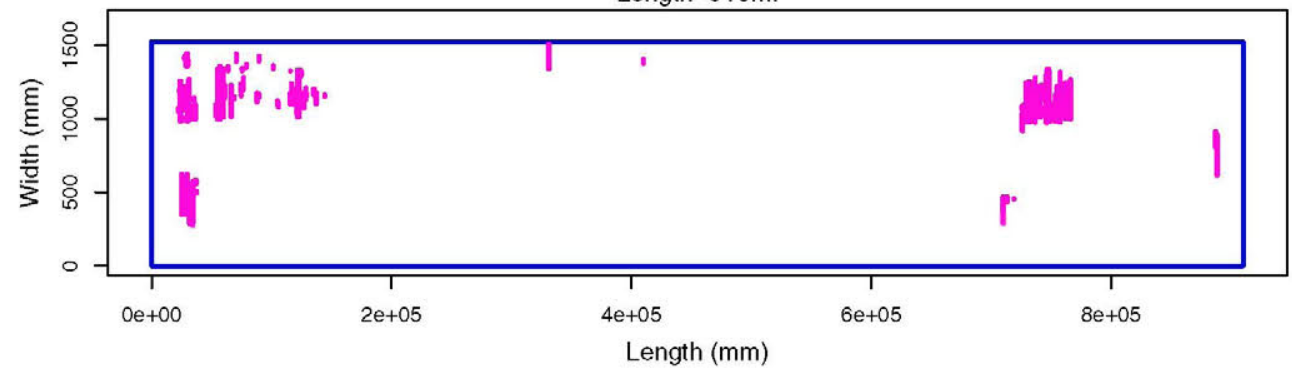

- Scale CC5 | Scale CC3 | Scale CC7

PICKLING LINE (Side 0)

Length $=880 \mathrm{~m}$.

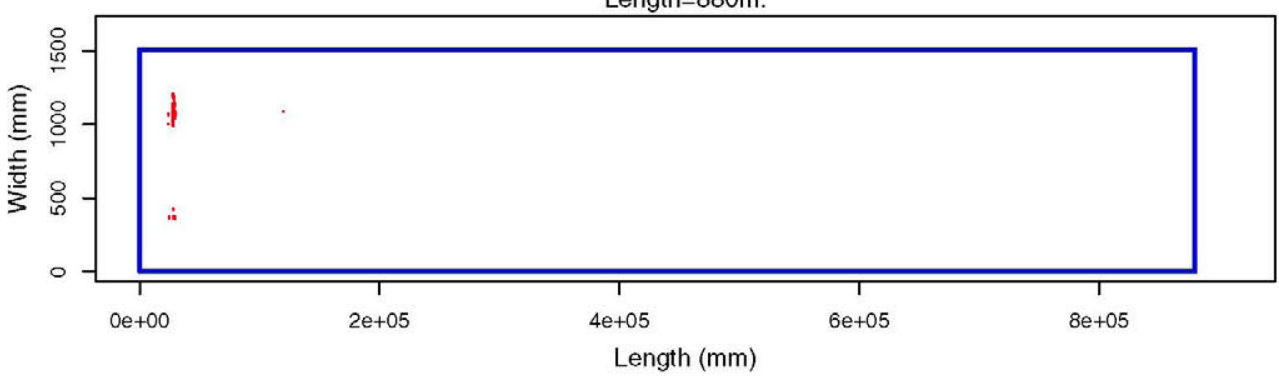


their results in order to be able to use them as online support for the surface inspector $[7,20]$. Today, there is an increasing desire for in-depth use of these results, although there is no general approach to handling the data and improving the utilization.

Similar problems arise in surface-mounted technology that is used extensively in the production of printed circuit boards due to the high level of miniaturization and the increase of density in electronic device integration. Some authors [5] have proposed intelligent systems over optical systems in order to evaluate the quality of joints.

In this case, the production process involves hot rolling technology for strip production, where some surface defects are created (see Fig. 2). Later, a pickling line is used for HCl-based surface cleaning. Then, if appropriate, the coil will be protected by means of an HDG process [4].

From here, various finishing processes are scheduled, according to the product's final destination. However, some additional strips need to be included in order to be able to downgrade any strips that do not fulfill surface quality criteria.

For other relevant strip quality parameters, such as mechanical properties, nonlinear statistic and nonparametric strategies, such as neural networks, have been used for online prediction of quality during the rolling or hot-dip galvanizing of coils [17, 23, 25]. These strategies contribute to decision-making processes at the operator level, reduce the production of scrap, or help to produce better products. Indeed, they have also been used to model a furnace during the production process [18] or to simply model steel properties [16].

Unfortunately, in regards to the state of the art, the approach for surface inspection is to classify the material, aided by the ASIS information and preclassification tools, but always at a local level [10]. Because of the sensitivity levels for ASIS as well as for several other factors, the strategy makes possible a very different quality assessment at different steps in the process for the same strip needs to be developed, mainly because of the difficulties faced by defect localization. Where position can be determined by a network of sensors, some techniques use a Gaussian distribution to explain localization probability, in such a way that each sensor can contribute to refining the final position [9]. In addition, [11] uses a similar approach with regards to the impact of a given surface defect. These basic ideas will be considered in the research presented here as a way to deal with position uncertainty of defects across facilities.

As the level of automation continues to rise, offering increasing efficiency, it also leads to more complex systems. Operating such systems requires reliable integration with the shop floor and higher levels, like manufacturing executives and the office, to enable process monitoring, quality control, and efficient maintenance. Systems must face this growing complexity, as well as their customers' demands for low acquisition and operating costs, adaptability to their needs, and improved service in order to keep up with increasing competition in a global market [1]. A modular way to deal with such complexity increment is to consider a service-oriented architectural framework for human task execution. It improves the execution by automating and semiautomating decision making based on agent technology. Over the last few years, a variety of agent platforms, like JADE [14], Grashopper [21], and Cougaar [15, 26], and standards like FIPA [12], have evolved that cover different aspects of agent programming, making them convenient to use at industrial level [2].

The complexity of these systems requires that advanced strategies and algorithms be considered in the decision-making process, allowing benefits to be obtained from the information hidden in the process data. Standards such as cross industry standard process for data mining (CRISP-DM), SEMMA, and PMML are making data mining processes easier. Nevertheless, most projects are being developed more as art than as science, making it difficult to understand, evaluate, and compare results as there is no standard methodology. A methodology based on RUP and CRISP-DM has been proposed [6] in order to address these weaknesses.

Techniques identified by the methodology have been used in recent times by those looking to promote the advance of manufacturing technology, like classification trees [8], or regression estimators based on neural networks and support vector machines [13].

A keystone prerequisite for adaptation is the ability to learn, a process based on knowledge discovery and growth. A way to reshape this concept is to examine a large volume of real-time manufacturing data collected during manufacturing operations and to use the insights gained to support decision making and adaptive process control. A natural way to do this is by using a rulesoriented approach [22].

All of these techniques that are combined in the previously introduced methodology will be considered in the present research in order to provide better support to the decision-making process in a multi-facility environment in a search for better defect management than obtained by considering isolated steps of production (see Fig. 4). In particular, support vector machines (SVM) will be considered to constitute an acceptable technique for regression models [3, 19]. However, some other activities will require other tools. For instance, 


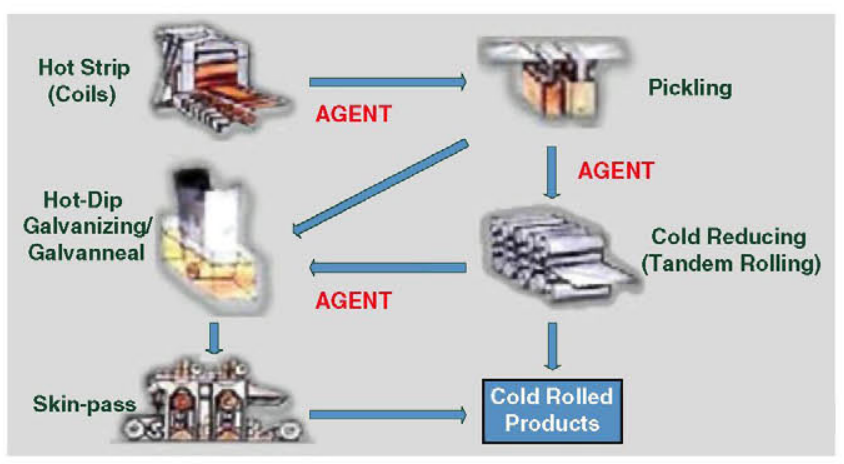

Fig. 4 Multi-agent system allowing a distributed decision-making approach

classification will be addressed by means of decision trees and J48 trees [24].

\section{Defect modeling}

The first problem to address is how to represent different types of defects on the product surface as their positions can change due to thermal or geometric variations.

Another aspect to consider is the accumulation of damage arising from different defects located closely together, as well as the different significance of different defects, according to the customer's quality criteria.

In accordance with what the literature review presented has suggested, the authors have chosen to use Gaussian functions. This makes it possible to deal with small defect position variations as well as adding their effect and that of those that are nearby. In fact, the paper suggests considering a probabilistic approach for severity instead of the exact position of the defect. In this way, general geometric transformations are applied to the defect's position, and, based on the estimated position, specific functions taking care of the severity are defined for different product regions. The estimation of defect evolution is now carried out in terms of region severity and its probability of survival to the final stage, depending on the type of operations being carried out. With this approach, it will be also possible to develop predictive estimates for a defect's evolution and its survival capability as well as its severity at different stages.

This paper proposes considering an axis that is perpendicular to the product to measure quality damage. In fact, due to the nature of this application, the authors propose to use a two-dimensional Gaussian function that represents each defect found. Its elementary formula is:

$f(x, y)=A * e^{-\left(a\left(x-x_{o}\right)^{2}+b\left(x-x_{o}\right)\left(y-y_{o}\right)+c\left(y-y_{o}\right)^{2}\right)}$

where the matrix $\left(\begin{array}{ll}a & b \\ b & c\end{array}\right)$ is positive definite.

For the general form of the equation, the coefficient $A$ is the amplitude and $\left(x_{o}, y_{o}\right)$ is the center of the blob. The lateral extension of defect influence is controlled by the variance along each axis.

Extension of this elementary approach to all defects in the coil will produce a function representing a defect's influence,

$$
\begin{aligned}
& \operatorname{defects}(x, y) \\
& =\sum_{i \in C_{\mathrm{D}}} \sum_{j=1}^{N_{i}} A_{i} * e^{-\left(a_{i}\left(x-x_{o i j}\right)^{2}+b_{i}\left(x-x_{o i j}\right)\left(y-y_{o i j}\right)+c_{i}\left(y-y_{o i j}\right)^{2}\right)},
\end{aligned}
$$

where $C_{\mathrm{D}}$ represents the set of defect classes of relevance in this particular problem and $N_{i}$ represents the number of defects classified as class $i$ that have been found in one particular product.

By integration, it is possible to qualify damage of the product as a whole,

$D e F($ product $)=\int_{0}^{L_{X}} \int_{0}^{L_{Y}} \operatorname{defects}(x, y) d x d y$,

where the size of the product is $L_{X}$ meters in length by $L_{Y}$ meters in width.

The proposed approach makes it possible to support different quality sensitivities just by changing parameter $A_{i}$ or variances along the axes according to the user 's interest or the relative relevance of each defect type.

After having introduced a method in which there is an interest in considering all types of defects at a glance, instead of considering each defect in an isolated fashion, the next step in seeking quality control improvement is a comparison of the defect maps from each facility involved in the transformation of the product.

Indeed, it is necessary to face the defect map comparison across facilities (see Fig. 5). In particular, this comparison introduces a new challenge that is related to the reliability of the global process itself.

Usually, surface defects are identified by specific sensors that are locally calibrated and maintained at scheduled time intervals. Taking into account the sequence for processing products, it should be possible to find facilities that are focused mainly on cleaning defects that have existed to that point. With these kinds of production systems, there is an additional interest in considering quality from a global point of view. Hence, 
Fig. 5 General 3D view of defect maps for the same coil at different facilities. Top is HSM map for side 0 , and bottom is PKL map for the same side
COIL CODE: I $1115034(0)$

Defect density 3D. $K(x)=100, K(y)=10$

HSM (Side 0)

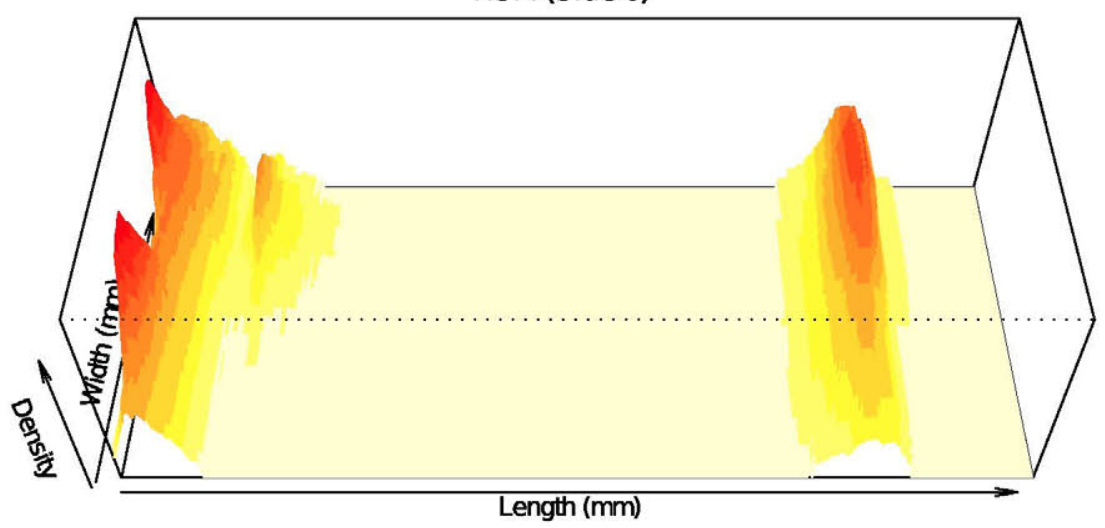

PICKLING LINE (Side 0)

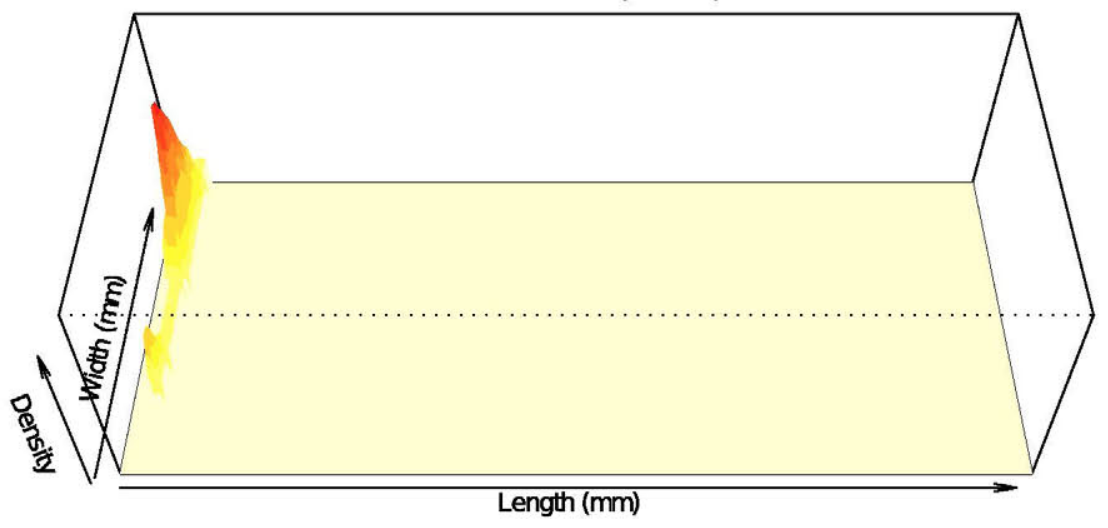

some level of intensity of defects can be acceptable as they will be removed in the facilities that follow.

Because of the modification of the geometry of the products at each facility, there is some uncertainty in defect position to be resolved. In this regards, a specific metric is required (see Fig. 6) in order to measure the impact of defects evolution.

In order to define the measure previously referred, let us designate the following:

$\Omega \quad$ as the set of portions of product

$\Sigma$ as the sigma-algebra over $\Omega$

$\mu$ as the area function, with domain as $\Sigma \otimes \Omega$ and verifying,

1. Non negativity

2. Null empty set means a zero value for its measure

3. Countable additively $\mu(\Omega, z)=\int_{\Omega} s(x . z) \quad d x$, where

$s(x, z)= \begin{cases}1 & \text { if } \operatorname{defects}(x)>\mathrm{z} \\ 0 & \text { otherwise }\end{cases}$

It is a straightforward exercise to verify that the function defined in Eq. 3.4 is monotonic and satisfies triangular inequality for every given $z$ value. So, these properties allow $\mu(\Omega, z)$ to be identified as a formal measure.

Thus, this metric makes it possible to identify the impact of specific production parameters or even assess the calibration of different sensors to a common understanding of the same intensity of damage.

As the surface defects are identified by an ASIS system, its sensibility and algorithms are calibrated locally and maintenance operations are scheduled for operating time periods. Taking into account the sequence for processing coils, which is the hot strip mill followed by the pickling line and then the hot-dip galvanizing 
Fig. 6 Isointensity of defect relevance according to the metrics chosen
COIL CODE: I1115034 (0)

Defect density 2D. $K(x)=100, K(y)=10$

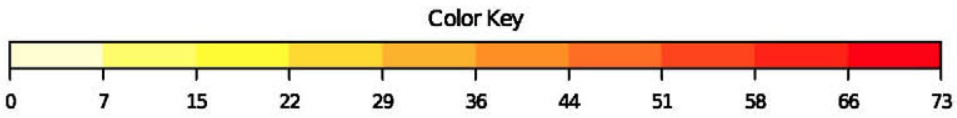

HSM (Side 0)

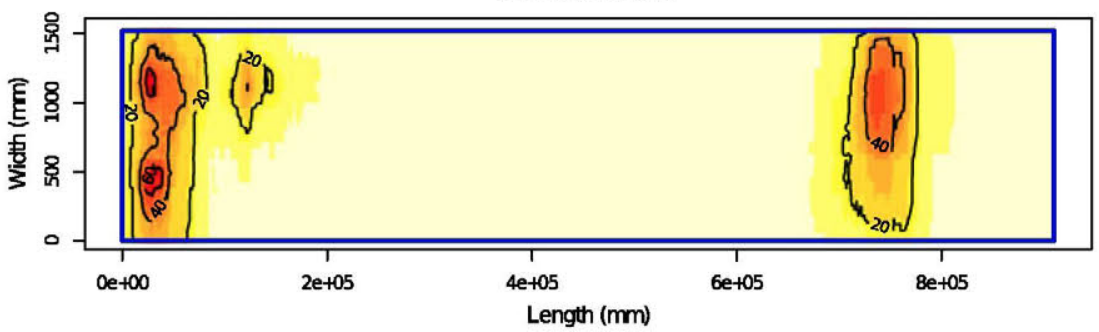

PICKLING LINE (Side 0)

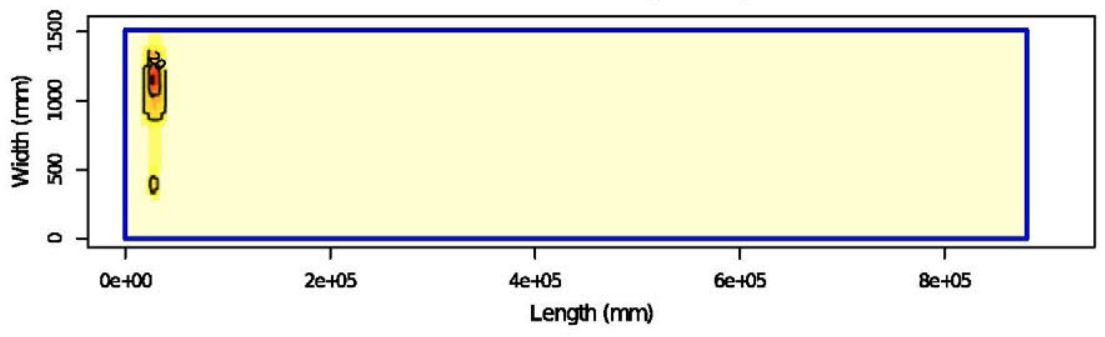

line, the second facility concentrates mainly on cleaning defects that have existed to that point in order to feed material that is good enough to the third facility. From this, it is clear that the pickling line is not concerned with new scale-related defects.

It is possible to take advantage of this knowledge and to accept an axiom, which is that scale-related defects are not created by the pickling line. This concept can be used initially on each coil of steel strip in order to measure the number of instances in which a defect that did not exist at the HSM is detected at the PKL. After a per coil measurement, it will be possible to establish a criterion considering the evolution of this measure against time and a confidence level. Then, when the criterion (level) is exceeded, maintenance and calibration will be required on the HSM ASIS, PKL ASIS, and/or HDG ASIS.

There is an interest in measuring the intersection of defect coil maps coming from the HSM and the PKL when pickling line defects are greater in number than those detected at the HSM. This means incoherence, which is the reason for the interest in monitoring the reliability level.

The criterion used is the cardinality of the following set:

$\Delta=\left\{\operatorname{coil} / \mu\left(\operatorname{coil}, z_{H S M}\right)<\mu\left(\right.\right.$ coil,$\left.\left.z_{P L}\right)\right\}$
The total effect of the previous operation can be seen in Fig. 7 where, as an example, the criterion established was the number of incoherent coils per month ( $\Delta$ per month). One such coil more or less indicates sufficient reliability. Between one and two is classified as alert.

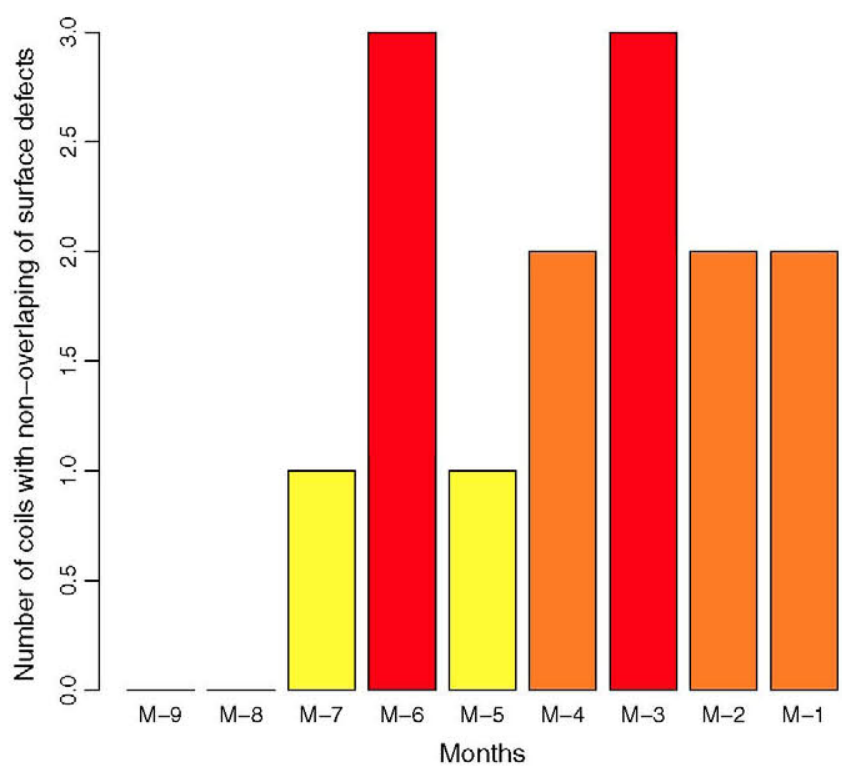

Fig. 7 Evolution of incoherences per month in a real environment. Relationship HSM vs PKL 
Three or more such coils signify unreliability and indicate that maintenance and calibration is required.

\section{Defect prediction}

The main interest in defect prediction is in developing a model that will predict the cleaning capability at the PKL (Fig. 8). The cleaning capability is measured as the percentage of affected surface, estimated according to Eq. 3.5. As long as there are coils that were processed previously by the existing methodology, it should be possible to create a model based on data, by supervised learning.

Variables considered in cleaning capability predictions include production parameters at the HSM-such as temperature and speed at different zones in the process and the number of passes through the reversing mill, etc.- as well as production parameters at the PKL (e.g., temperatures and concentrations of the bath, speed, etc).

According to the literature review, SVM technology is adequate for this particular application. The basic idea in SVM is to construct a simple linear decision surface in the high-dimensional feature space nonlinearly related to the input space. SVM maps the input data in a high-dimensional feature space by means of a kernel function, i.e., a function returning the inner product between two points in a suitable feature space, thus defining a notion of similarity, with little computational cost even in very high-dimensional spaces.

The learning then takes place in the feature space, and the data points appear only inside scalar products with other points. This is often referred to as the kernel trick.

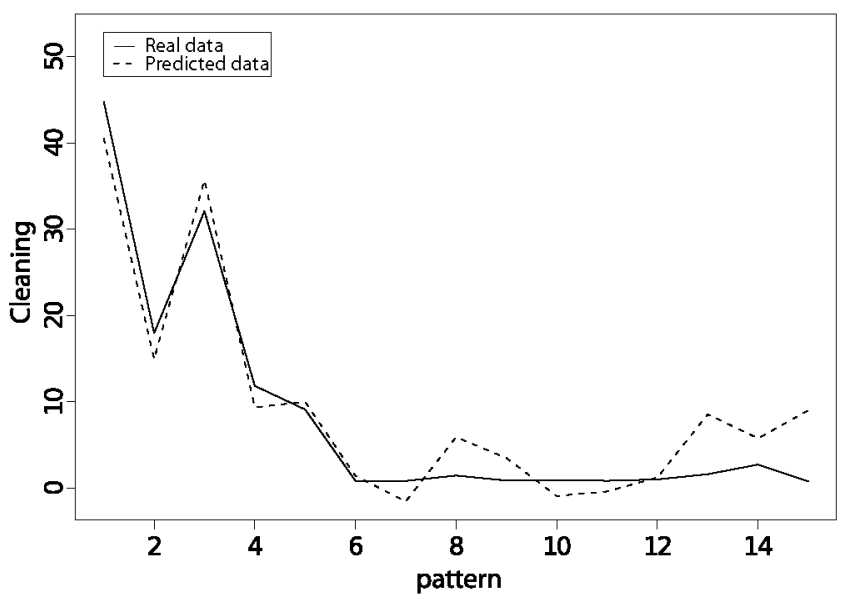

Fig. 8 Percentage of cleaning at PKL according to developed SVM model against real data not known by the model
Kernels commonly used with SVM include the following:

- The Gaussian and Laplace radial-basis function (RBF) and Bessel kernels, which are generalpurpose kernels that are used when there is no prior knowledge about the data.

- The linear kernel, which is useful when dealing with large sparse data vectors as is usually the case in text categorization.

- The polynomial kernel, which is popular in image processing, and the sigmoid kernel, which is used mainly as a proxy for neural networks.

- The splines and ANOVA RBF kernels, which typically perform well in regression problems.

The performance of a selected model-with an ANOVA RBF kernel-was measured for every coil available in each data set (training and pure testing data sets). The following information is shown (see Table 1):

- Root mean square error (RMSE). To calculate the RMSE, the individual test errors are squared, added together, and divided by the number of individual errors. Then, the square root of the foregoing (the mean of the squared errors) is calculated. This gives a single number that summarizes the overall error.

- Normalized root mean squared error (NRMSE). This is the RMSE divided by the range of observed values. This value is often expressed as a percentage, with lower values indicating less residual variance than high values.

- Minimum and maximum error. The minimum and maximum errors indicate the range of model prediction errors.

- Mean error. This is the mean value of the prediction errors.

- Standard deviation (SD). This is a measure of the dispersion of errors and indicates the variability of these errors from the mean. If the value is small, the errors are close to the mean. If the value is high, they are dispersed farther from the mean.

A direct lecture shows that an ANOVA-type kernel performs better than a Gaussian one, because the NRMSE is lower both for training and for test processes. Also, the SD is lower. This result is coherent with a previous comment in this paper about ANOVA kernels being very convenient for regression problems.

The impact of such kinds of global models (understood as models across facilities) is that it becomes possible to estimate at the HSM stage what will probably exit the PKL. With this knowledge, the decision whether to process, to downgrade, or to reroute the 
Table 1 Errors for training process (the top two lines) and for testing (the two bottom lines)

\begin{tabular}{lllllll}
\hline Kernel RBF & RMSE & NRMSE (\%) & Min. error & Max. error & Mean error & SD \\
\hline Gaussian & 1.4 & 1.68 & -1.72 & 1.72 & -0.065 & 1.41 \\
ANOVA & 1.3 & 1.56 & -1.96 & 2.03 & 0.040 & 1.31 \\
Gaussian & 3.74 & 8.50 & -8.24 & 4.27 & -1.02 & 3.73 \\
ANOVA & 2.80 & 6.35 & -7.17 & 3.69 & -0.47 & 2.85 \\
\hline
\end{tabular}

process can be made in a much more consistent way. After gathering this information, the multi-agent system that is implemented produces a sensitivity analysis of other factors, such as the concentration of chemicals in bath tanks and the temperature in these tanks or whatever technical factor appears to be relevant in the facility. The HSM agent will be able to determine the probability that the current coil will be cleaned by the PKL with its current setup, or with the setup changed slightly. This additional capability is being used, from a technical point of view, to establish acceptable conditions at the PKL and to attempt to find a balance between line capacity and line efficiency.

Alternatively, the HSM agent will recommend that the coil be downgraded at this point by removing it from the current order.

Even more relevant is the ability to investigate complex relationships like defect density against different relevant variables, even according to the grade of steel. Specific tools like decision trees and J48 trees were used, and different rules were validated (see Fig. 9).

In this case, significant variables were described in Table 2. In this case, the precision of the tree is $82 \%$ for the TRUE class and $86 \%$ for FALSE class with an ROC area of 0.865 , which allow us to consider the classifier as valuable.

In order to assess the models that have been oriented to improving the decision strategy depending on surface quality, three different versions of these models have been developed. A priority was being able to explain to users the main reasons for the results.
This meant that a tree reasoning-based technology was required at the first level.

The three different versions were:

1. A complete model that takes into account process parameters coming from the HSM and the PKL. This model can be run once the coil has been pickled and if, for example, some scale remains and the quality managers want to determine the reasons for this.

2. A simplified model that, takes into account mainly parameters that come from the HSM. The only PKL variable that is considered is the pickling speed. For this speed, a high value, close to the maximum line speed, is assumed. This model can be used to predict, when the strip is at the exit of the HSM, whether the pickling speed needs to be reduced to ensure receiving the desired quality of the pickled coil.

3. A simplified model, with parameters coming only from the HSM. In this case, a low value for the pickling speed, close to the speed used for problematic coils, is assumed. This model can be used to predict, already at the exit of the HSM, if it would be useful to reduce the pickling speed to ensure the correct quality of the pickled coil.

Figure 10 shows the results of the complete model for a specific coil. The bar on the left represents the probability of having scale after pickling (56\% in this case). Each line gives information about the contribution of a parameter taking into account the color gradient from
Fig. 9 J48 tree explaining high density of defects according to different variables relevant to one particular steel grade

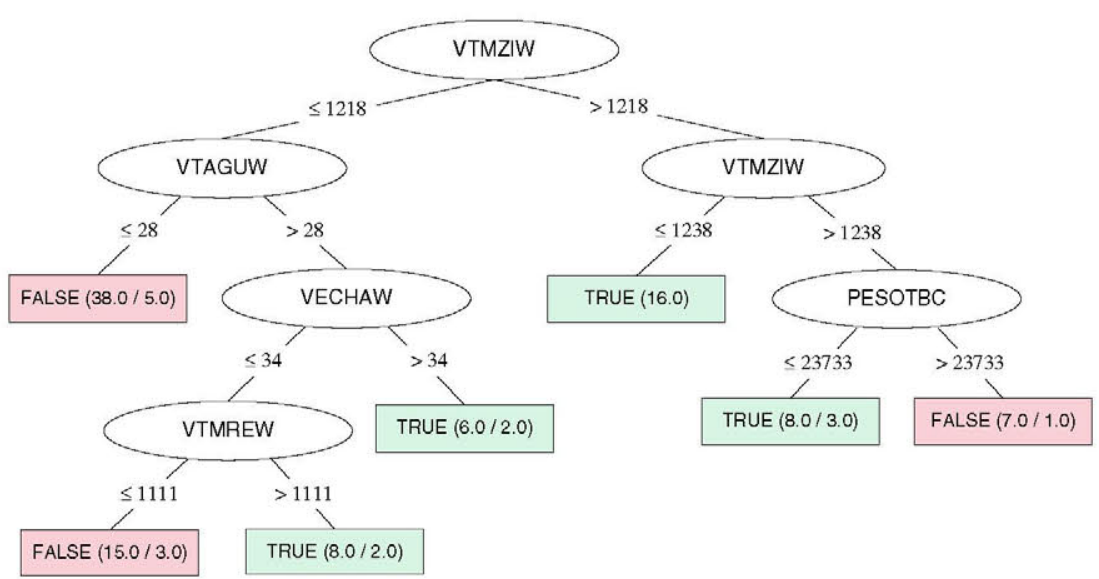


Table 2 Variable meanings that explain high defect levels

\begin{tabular}{ll}
\hline Name & Meaning \\
\hline VTMZIW & Temperature for the furnace equalization zone \\
VECHAW & Thickness of slab \\
PESOTBC & Weight of slab \\
VTMREW & Average temperature at finishing mill \\
VTAGUW & Temperature for cooling water
\end{tabular}

green (parameters have a value that tends to avoid scale) to red (the values of the parameters are negative for the scale defect). For example, the text that appears in the first line means the following:

[SM01] FIM_TIJ_TMP $=1107.2 \geq 1079.4$

- SM01: The parameter belongs to the HSM (the parameters from the pickling line start with [DP02]). This is an internal code used by the company.

- FIM_TIJ_TMP: The name of the specific parameter (temperature at the entry of the finishing mill in this case).

- 1107.2: The real value of the parameter considered for this coil.

- 1079.4: The threshold to which the real value is compared.

In this way, operators know that one way to have a lower defect risk is to reduce the entry temperature to a value below the limit proposed by the model.

On this specific coil, some small scale defects were present after pickling. Model results can be considered to be correct in this case.

In summary, the operating quality system was moved from local decisions to a more global basis, taking into account additional information provided by other agents. This makes it possible to add information about process parameters in order to learn the conditions

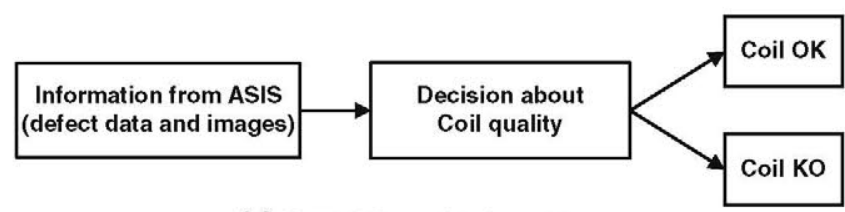

(a) Initial flow of information

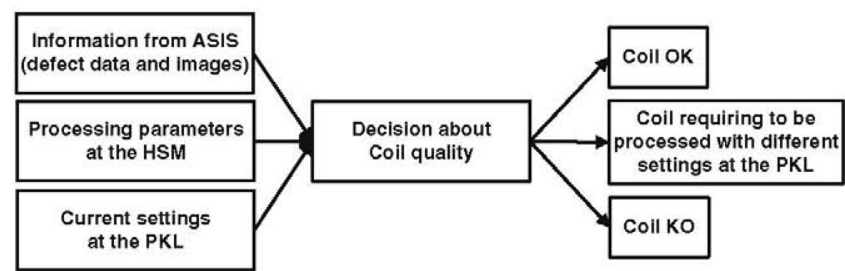

(b) Strategy with the multiagent system helping the decision maker.

Fig. 11 Operating strategy for making a decision regarding its quality at the exit of HSM

under which a defect was produced and, by the use of the adequate models to attempt to predict how easily scale can be removed (see Fig. 11b).

\section{Conclusions}

The focus of the work presented here was to apply some intelligence to the ASIS system in order to help operators to obtain better classification than the existing one that relies on local criteria. This paper proposes a global function to consider the influence of defects on the strip.

When it is applied to one strip by using different sensitivity criteria, the proposed approach makes it possible to identify valuable strip areas. This becomes useful for operators who obtain additional information
Fig. 10 Rules from complete version of the model used for practical implementation

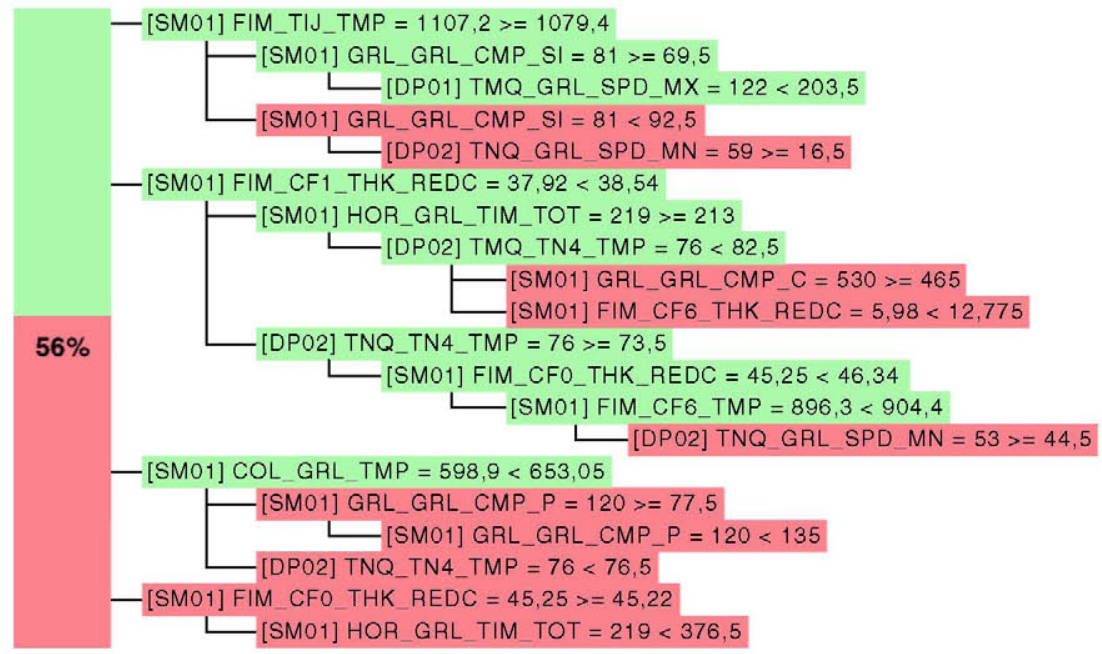


Fig. 12 ROC curve of the advanced decision tree model implemented and tested in the plant

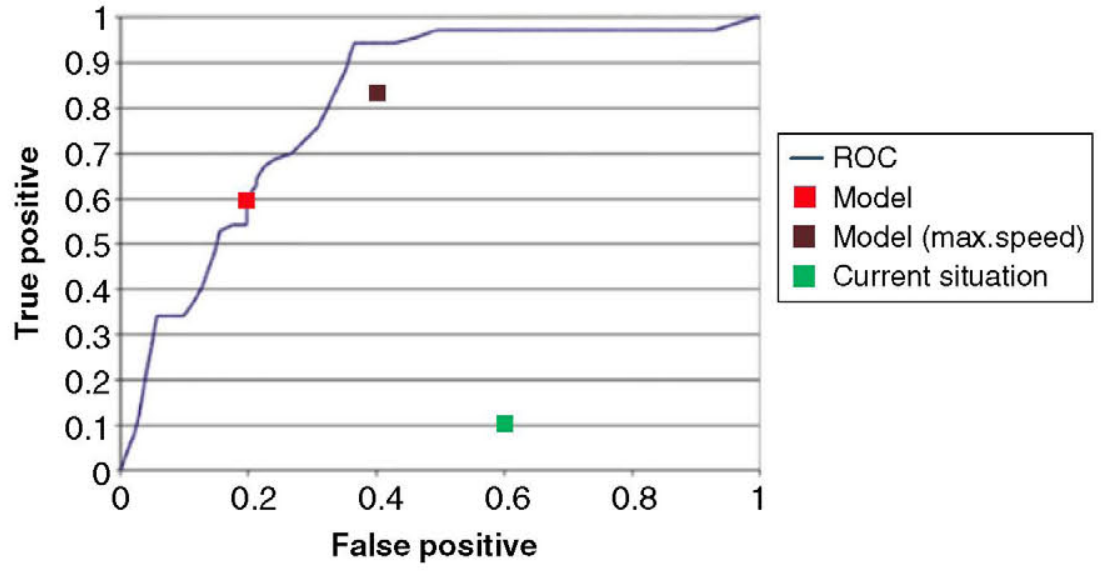

for cutting the strip appropriately and ensuring that the customer's order becomes as complete as possible.

The use of software multi-agent technology makes for more clear functionality by causing independent activities to be handled by different agents.

Additional potentialities of the proposed strategy are the establishment of a relationship between operating parameters of facilities and defect levels, as well as the current operating status of a particular facility. This can be the first step in the early rerouting of products (strips after the HSM in the presented case study) or processes or in predicting the need for quality downgrading of a particular product. In turn, this leads to better estimates of the number of products required to fully complete an order.

The presented strategy and models have been implemented in the plant and the results will be tracked for several months.

Figure 12 is an attempt to summarize the results. The green point represents the old situation. It can be seen that the performances of previously used criteria is very poor. More than half of the coils that are downgraded at the HSM because of scale defects are correctly cleaned during pickling. On the other hand, only about $10 \%$ of coils with scale after pickling are correctly predicted at the hot strip mill.

The ROC curve built with validation data shows that, although the model is not perfect, it is able to improve clearly the current situation. The red point represents the values for a large number of coils processed under completely normal production conditions. The point lies exactly on the ROC curve, which means that the model is robust and its performances does not decline when used industrially.

The brown point represents the results of the model applied at the exit of the HSM assuming a maximum speed at the pickling line. In this case, we adopt a secure position, asking more often for a reduction of the speed of the pickling line. It can be seen that, if the recommendations are followed, scale could be avoided on more than $80 \%$ of coils that presently have scale after pickling. The negative part is that this speed reduction would not be necessary in about $40 \%$ of the cases. The point for this model lies under the ROC curve.

This is normal, as the model is applied at the exit of the HSM, and not all of the parameters are known at present.

This paper outlines one procedure for making analytical comparison of damaged product (a coil's surface in the case study presented), after being processed in different facilities, with special consideration to physical transformations due to product transformations (a coil's different temperatures). The affected surface is estimated by a model that considers additive local effects. Also, a function providing a global damage value for the product is introduced. A comparison of the same coil between facilities by means of this function makes it possible to measure successful production capability for the entire process at specific operating conditions.

The proposed approach makes it possible to use the same technology for the same product at different facilities and to define a reliability measure for the global process chain, which is a relevant innovation for different sectors like the steel industry.

\footnotetext{
Acknowledgements The authors especially thank the referees for their valuable contributions to the improvement the final version of this paper. This work has been partially supported by the EU RFCS research project IRSIS (code RFSR-CT-200600036), as well as by the Spanish national research grant Salvador de Madariaga code 2010-0014. For these reasons, the authors express their personal appreciation to the European Commission for its support and, in particular, to the RFCS program and the Spanish Ministerio de Educación.
} 


\section{References}

1. Abellán-Nebot J, Romero Subirón F, Serrano Mira J (2012) Manufacturing variation models in multi-station machining systems. Int J Adv Manuf Technol 1-21. doi:10.1007/ s00170-012-4016-4

2. Ameri F, McArthur C (2012) A multi-agent system for autonomous supply chain configuration. Int J Adv Manuf Technol 1-16. doi:10.1007/s00170-012-4392-9

3. Bishop C (2005) Pattern recognition and machine learning. Springer

4. Bolt PH (2004) Understanding the properties of oxide scales on hot rolled steel strip. Steel Res Int 75(6):399-404

5. Giaquinto A, Fornarelli G, Brunetti G, Acciani G (2009) A neurofuzzy method for the evaluation of soldering global quality index. IEEE Trans Ind Inf 5(1):56-66

6. González-Aranda P, Menasalvas E, Millán S, Ruiz C, Segovia J (2008) Towards a methodology for data mining project development: the importance of abstraction. In: Lin T, Xie Y, Wasilewska A, Liau CJ (eds) Data mining: foundations and practice, studies in computational intelligence, vol 118. Springer Berlin / Heidelberg, pp 165-178

7. Henry D, Permane P, Dubuisson Y (1992) Artificial vision. detection and filling of surface defects on pickled sheet. Rev Métall 89(7-8):645-654

8. Jämsä-Jounela SL, Tikkala VM, Zakharov A, Pozo Garcia O, Laavi H, Myller T, Kulomaa T, Hämäläinen V (2012) Outline of a fault diagnosis system for a large-scale board machine. Int J Adv Manuf Technol 1-15. doi:10.1007/ s00170-012-4296-8

9. Jeong H, Lee JS, Bae SM (2011) Defect detection and localization in plates using a lamb wave time reversal technique. Int J Precis Eng Manuf 12:427-434. doi:10.1007/ s12541-011-0055-2

10. Kang GW, Liu HB (2005) Surface defects inspection of cold rolled strips based on neural network. In: Proceedings of 2005 international conference on machine learning and cybernetics, vol 8, pp 5034-5037

11. Lacasse L, Tahan A, Liu Z (2008) Robust method for the automatic characterization of a wooden board's geometrical state. Qual Eng 20(2):221-231. doi:10.1080/ 08982110701733042

12. Levy R, Chen W, Lyell M (2009) Software agent-based framework supporting autonomous and collaborative sensor utilization (aamsrt). In: Eighth international conference on autonomous agents and multiagent systems. Budapest, Hungary $10-15$ May 2009

13. Masood I, Hassan A (2012) Pattern recognition for bivariate process mean shifts using feature-based artificial neural network. Int J Adv Manuf Technol 1-18. doi:10.1007/ s00170-012-4399-2

14. Mosqueira-Rey E, Alonso-Betanzos A, Guijarro-Berdinas B, Alonso-Rios D, Lago-Pineiro J (2009) A snort-based agent for a JADE multi-agent intrusion detection system. Int $\mathrm{J}$ Intell Inf Database Syst 3(1):107-121

15. Omicini A, Ricci A, Viroli M (2008) Artifacts in the A\&A meta-model for multi-agent systems. Auton Agent MultiAgent Sys 17(3):432-456

16. Ordieres-Meré J, de Pisón-Ascacibar FJM, González-Marcos A, Ortiz-Marcos I (2010) Comparison of models created for the prediction of the mechanical properties of galvanized steel coils. J Intell Manuf 21(4):403-421

17. Ordieres-Meré JB, González-Marcos A, González JA, Lobato-Rubio V (2004) Estimation of mechanical properties of steel strips in hot dip galvanizing lines. Ironmak Steelmak 31(1):43-50

18. Pernía-Espinoza A, Castejón-Limas M, González-Marcos A, Lobato-Rubio V (2005) Steel annealing furnace robust neural network model. Ironmak Steelmak 32(5):418-426

19. Schökopf B, Smola A (2001) Learning with kernels: support vector machines, regularization, optimization, and beyond. The MIT

20. Sun H, Xu K, Xu JW (2003) Online application of automatic surface quality inspection system to finishing line of cold rolled strips. J Univ Sci Technol Beijing 10(4): 38-41

21. Tosic M, Zaslavsky A (2005) The external fault-tolerant layer supporting multi-agent systems from different vendors. In: IEEE/WIC/ACM international conference on intelligent agent technology

22. Žapčević S, Butala P (2012) Adaptive process control based on a self-learning mechanism in autonomous manufacturing systems. Int J Adv Manuf Technol 1-19. doi:10.1007/ s00170-012-4453-0

23. Wang W, Hong G, Wong Y, Zhu K (2007) Sensor fusion for online tool condition monitoring in milling. Int J Prod Res 45(21):5095-5116

24. Witten IH, E Frank MH (2011) Data mining, practical machine learning tools and techniques with java implementations. Kaufmann Publishers

25. Zheng H, Gong DY, Wang GD, Liu XH, Zhang PJ (2007) Software of predicting mechanical properties of strip steel by using bp networks. J Iron Steel Res 19(7):54-62

26. Zinky J, Shapiro R, Siracuse S, Wright T (2005) Implementing QoS-adaptation in coordination artifacts by enhancing Cougaar multi-agent middleware. In: 2nd IEEE symposium on multi-agent security and survivability (MASS) 